\title{
Climate past and future
}

\author{
Earth's climate is changing rapidly. A closer look at the planet's distant past can help determine its \\ sensitivity to changes in atmospheric greenhouse gas concentrations.
}

Two principal approaches are used to help constrain future climate. First, the laws of fluid dynamics, moulded into climate model source code, provide a theoretical baseline for understanding changes in ice, ocean and atmosphere. The principles of physics, supplemented with about a hundred years of direct observations, allow an estimate of what will happen when atmospheric greenhouse gas concentrations rise beyond the range of instrumentally recorded history. Second, palaeoclimate researchers are increasingly pursuing a complementary approach. Natural archives in ice, rocks or sediments harbour information about climate conditions and atmospheric composition of the distant past. Understanding how the Earth fared during earlier episodes of soaring greenhouse gas concentrations or sudden change can yield insight into the range of possible future responses.

Three Commentaries in this issue (pages 414-420) outline how a better grasp of past climate variability can be achieved, and how it will help with projections of future change. As discussed in two of the pieces, once proxybased climate and $\mathrm{CO}_{2}$ reconstructions satisfy tighter quality control, they will reveal Earth's past sensitivity to greenhouse gas forcing. The third article argues that state-of-the-art complex climate models could be too stable and need more thorough validation against documented sudden changes in the past.

The latter observation broadly follows on from a problem posed by the project climateprediction.net (http:// climateprediction.net/). Distributed computing - using idle time on volunteers' computers - allowed the project to run a grand ensemble of more than 2,000 unique variants of a global climate model, all able to reproduce twentieth-century observations. The result was startling: warming in response to a doubling of atmospheric $\mathrm{CO}_{2}$ concentrations varied between model variants over the huge range of 2 to $11^{\circ} \mathrm{C}$ (Nature 433, 403-406; 2005), much broader than the range of 2.1 to $4.4^{\circ} \mathrm{C}$ covered by the climate models that are cited in the assessments of the Intergovernmental Panel on Climate Change (IPCC). These results, too, hint that the IPCC-type models may underestimate the amplitude of the climate's response to a change in boundary conditions.

Detailed comparisons of a range of model variants with past climate reconstructions could help narrow down the possibilities. Some models whose results are perfectly compatible with the twentieth-century record may not capture past warm periods or instances of sudden change, and could thus be ruled out.
But first we must work on our understanding of what happened, and how rapidly (see also page 481 in this issue). To this end, reconstructions of climate variables such as temperature, together with external influences such as atmospheric composition, are essential. Once the details of past events are documented, they can provide a desperately needed benchmark for climate models.

It will take a concerted effort to derive a suite of robust palaeoclimatic proxies that can shed light on the sensitivity of the climate system to changes in the planet's make-up. And a large injection of modellers' ingenuity will be required if currentgeneration climate models are to be tested on extended periods in the past: complex models can occupy a supercomputer for months, just to simulate a period of one hundred years.

Availability of state-of-the-art climate models that are capable of recreating the climate dynamics and conditions of past warm and glacial periods, as well as the more stable twentieth-century conditions, would bring a step change in our understanding of what is to come over the next century or two. In combination, climate models and past analogues have the potential to deliver such progress.

\section{Think like a journalist}

\section{A cultural divide separates science from the media. To bridge the gulf, Nature Geoscience presents a science writer's perspective on the Earth sciences in a new monthly column.}

Broad exposure in the press, if accurate, is good for science. For an individual researcher, however, interactions with the media are not a straightforward road to prestige. Handled badly, an interview can equally lead to colleagues' raised eyebrows (or worse). Demand for informed comment on geoscience topics is on the rise, with climate change, natural disasters and environmentally damaging accidents increasingly in the public eye. The more Earth scientists understand how the media works, the more likely they are to be able to inject the latest research into the public debate.

To aid communication between science and the media, Nature Geoscience is launching a monthly column by geologist and science writer Axel Bojanowski. The first instalment can be found on page 421 of this issue. Each month, Bojanowski will explore an Earth science story that has garnered press attention. In an accompanying box, he will explain what it was about the science that captured his attention as a journalist, and how the story was received by the audience. Bojanowski has been covering all things geoscience for almost fifteen years, and currently writes for Spiegel Online, the leading Germanlanguage news portal.

First and foremost, the column is intended to entertain. But we hope that the accompanying box will additionally offer insights into the transformation of a scientific paper into a piece of news. A journalist's viewpoint of a popular news story should complement and perhaps challenge our readers' more habitual roles as scientists and consumers of news. We hope that some familiarity with the newsmaking process will help our readers to communicate their perspective more efficiently when the occasion arises.

It is important to us that the geosciences are reported accurately in the mainstream media. To that end, Earth scientists must know how journalists work - and work with them when called upon. 